\title{
Solving surface plasmon resonances and near field in metallic nanostructures: Green's matrix method and its applications
}

\author{
GU Ying ${ }^{1}$, LI Jia ${ }^{1}$, MARTIN Olivier J F² \& GONG QiHuang ${ }^{1 *}$ \\ ${ }^{1}$ State Key Laboratory for Mesoscopic Physics, Department of Physics, Peking University, Beijing 100871, China; \\ ${ }^{2}$ Nanophotonics and Metrology Laboratory, Swiss Federal Institute of Technology Lausanne (EPFL), CH-1015 Lausanne, Switzerland
}

Received March 24, 2010; accepted May 5, 2010

\begin{abstract}
With the development of nanotechnology, many new optical phenomena in nanoscale have been demonstrated. Through the coupling of optical waves and collective oscillations of free electrons in metallic nanostructures, surface plasmon polaritons can be excited accompanying a strong near field enhancement that decays in a subwavelength scale, which have potential applications in the surface-enhanced Raman scattering, biosensor, optical communication, solar cells, and nonlinear optical frequency mixing. In the present article, we review the Green's matrix method for solving the surface plasmon resonances and near field in arbitrarily shaped nanostructures and in binary metallic nanostructures. Using this method, we design the plasmonic nanostructures whose resonances are tunable from the visible to near-infrared, study the interplay of plasmon resonances, and propose a new way to control plasmonic resonances in binary metallic nanostructures.
\end{abstract}

nanooptics, surface plasmon resonance, near field, metallic nanostructure

Citation: $\quad \mathrm{Gu} \mathrm{Y,} \mathrm{Li} \mathrm{J,} \mathrm{Martin} \mathrm{O} \mathrm{J} \mathrm{F,} \mathrm{et} \mathrm{al.} \mathrm{Solving} \mathrm{surface} \mathrm{plasmon} \mathrm{resonances} \mathrm{and} \mathrm{near} \mathrm{field} \mathrm{in} \mathrm{metallic} \mathrm{nanostructures:} \mathrm{Green's} \mathrm{matrix} \mathrm{method} \mathrm{and} \mathrm{its} \mathrm{applications.}$ Chinese Sci Bull, 2010, 55: 2608-2617, doi: 10.1007/s11434-010-4023-5

Near field concerns the evanescent wave bound to a nanostructured material surface that decays exponentially within a subwavelength [1,2]. At the metallic-dielectric interface or in the isolated metallic nanostructure, due to the existence of collective oscillations of free electrons, surface plasmons [3-5] are excited and are accompanied with the enhancement of optical near field. The large near field at the surface comes from the electric field carried by the metal ( $\varepsilon_{d} E_{d \perp}=\varepsilon_{m} E_{m \perp}$ ), which is optimized by a proper matching of the geometry, the incident light, the metallic electric permittivity and dielectric environment. Nanostructures of noble metals strongly scatter and absorb light when the surface plasmon resonance (SPR) is excited [6]. The resonance frequency and intensity are dominated by the distribution of the polarization charge across the nanostructure [7]. This resonant enhancement has promoted many important applications, such as surface-enhanced Raman scattering [8],

*Corresponding author (email: qhgong@pku.edu.cn) biosensors and nanometer plasmonic waveguides [9-12], optical antennas [13], solar cells [14,15], nonlinear optical frequency mixing [16-18] and so on.

Currently, we can see a rapidly expanding array of metallic nanostructures. With the development of nanofabrication and nanolithography techniques, various metallic nanoparticles, such as nanospheres, nanoshells, nanorices, nanorings, nanostars, nanocages and nanotriangles, have been successfully fabricated [9]. For the nanostructures smaller than the electron mean-free path of the bulk metal, the dielectric permittivity becomes position-dependent. Generally, when the scale of nanostructure is larger than 10 $\mathrm{nm}$, dielectric permittivity is approximately looked as position-independent. Nanorods and nanostrips have attracted particular attention because the longitudinal plasmon absorption bands are tunable with their aspect ratio changing from visible to near-infrared [19-21]. Xia and coworkers have managed to synthesize 100-nm-length nanobars and studied their scattering spectra [22]. Due to the adjustability 
in geometry, metallic nanostrips can be used to assemble various plasmonic nanoparticle-based devices [13,21]. By controlling the core-shell ratios of nanoshells, resonances are tuned from the visible to infrared [9]. The nanocrescents have shown multiple, adjustable resonances and strong, enhanced near field due to their unique structure with tips and ring [23]. Simultaneously, based on the near field coupling among nanoparticles, red and blue shifts of resonances have been reported [24-27] as well as the scaling rules of these shifts $[28,29]$. The interplay of SPRs within metallic nanoparticles with different material parameters has been studied [30]. In addition to using the geometry and arrangement of nanostructures to adjust resonances, the use of their material parameters to control their plasmonic properties has also been proposed [31]. In particular, when both kinds of nanostructures are metallic, their plasmonic properties are greatly modified due to the existence of free electrons in the neighboring metal.

There are several ways to solve the SPR of metallic nanostructure, including the finite-difference time domain method (FDTD) [32], discrete dipole approximation (DDA) [33], Mie theory [34], Green's tensor method [35], the modified hybrid model [36] and spectral representation [37]. For FDTD, DDA and Green's tensor method, with given geometrical and material parameters, the information concerning optical near field can be calculated. Next, by scanning a wide wavelength region, their absorption and extinction peaks can be obtained. Mie theory and the modified hybrid model are generally used to deal with the sphere and spherical symmetry structure. Finally, the spectral representation is unlikely to spread over an arbitrarily shaped structure due to its quite complex integral form. By combining the Green's tensor method with Green's function formalism in the quasistatic limit [38], we have developed the Green's matrix method (GMM) to deal with the surface plasmon resonance and near field in the arbitrarily shaped subwavelength metallic structures and in binary metallic nanostructures [30,39]. The core of the Green's matrix method is to solve the eigenvalues and eigenvectors of Green's matrix, and these eigenvalues and eigenvectors correspond directly to the resonant properties of the system. The method provides a powerful tool for plasmonic optics. In the following, we will review the Green's matrix method, and its applications in the plasmonic resonance control and functional nanostructure design.

\section{Green's matrix method}

In this section, the salient properties of Green's matrix method in an arbitrarily shaped metallic cluster and in binary metallic nanostrcutures are reviewed [30,39]. Consider an arbitrarily shaped subwavelength structure with the electric permittivity $\varepsilon(\boldsymbol{r}, \omega)$ embedded in the homogeneous bulk material $\varepsilon_{0}(\omega)$. These subwavelength clusters are not neces- sarily inter-connected, but the tensor $\varepsilon(\boldsymbol{r}, \omega)\left(=\varepsilon(\boldsymbol{r}, \omega)-\varepsilon_{0}(\omega)\right)$ vanishes outside the clusters. The electric permittivity $\varepsilon(\boldsymbol{r}, \omega)$ is complex and frequency dependent in real metal. If a monochromatic incident field $E^{0}(\boldsymbol{r})$ (with the usual $\mathrm{e}^{-i w t}$ time dependence throughout this paper) impinges on the system, without any approximations in the Maxwell equations, the scattered field $E(\boldsymbol{r})$ is a solution of the propagation equation:

$$
-\nabla \times \nabla \times E(\boldsymbol{r})+k^{2} \varepsilon_{0}(\omega) E(\boldsymbol{r})+k^{2} \varepsilon_{s}(\boldsymbol{r}, \omega) E(\boldsymbol{r})=0,
$$

where $k$ is the vacuum wavenumber. With Green's tensor in a three dimensional system [40]

$$
\begin{aligned}
G^{0}\left(r, r^{\prime}, \omega\right)= & \left(\boldsymbol{I}-\frac{1-i k_{0} R}{k_{0}^{2} R^{2}} \boldsymbol{I}-\frac{-3+3 i k_{0} R+k_{0}^{2} R^{2}}{k_{0}^{2} R^{4}} \boldsymbol{R} \boldsymbol{R}\right) \\
& \times \frac{\exp \left[i k_{0} R\right]}{4 \pi R},
\end{aligned}
$$

where $R=|\boldsymbol{R}|=\left|r-r^{\prime}\right|$ and $k_{0}^{2}=k^{2} \varepsilon_{0}(\omega)$, the field $E(\boldsymbol{r})$ at any point $\boldsymbol{r}$ satisfies the Lippmann-Schwinger equation $[35,41]$ :

$$
E(\boldsymbol{r})=E^{0}(\boldsymbol{r})+k^{2} \int_{V} \mathrm{~d} \boldsymbol{r}^{\prime} \boldsymbol{G}^{0}\left(\boldsymbol{r}, \boldsymbol{r}^{\prime}, \omega\right) \varepsilon_{s}\left(\boldsymbol{r}^{\prime}, \omega\right) \cdot E\left(\boldsymbol{r}^{\prime}\right),
$$

where $V$ denotes the clusters subspace. The replacement of the traditional boundary conditions with the self-consistent coupling of all source points makes the Green's tensor method a powerful tool in dealing with various geometrically complex and subwavelength materials [1].

If the clusters subspace $V$ is divided into $N$ pieces of the volume $\delta V$ (with $\delta V<<\mathrm{V}$ ), eq. (3) becomes [39]

$$
\sum_{r^{\prime} \in V}\left[\varepsilon_{s}(\boldsymbol{r}, \omega) \tilde{G}^{0}\left(r, r^{\prime}, \omega\right)-\delta_{r, r^{\prime}}\right] \tilde{E}(r)=-\tilde{E}^{0}(r)
$$

with $\tilde{G}^{0}\left(\boldsymbol{r}, \boldsymbol{r}^{\prime}, \omega\right)=\delta V k^{2} G^{0}\left(\boldsymbol{r}, \boldsymbol{r}^{\prime}, \omega\right)$ and within the subspace $\varepsilon_{s}(\boldsymbol{r}, \omega)=\varepsilon_{s}\left(\boldsymbol{r}^{\prime}, \omega\right)$. This may be rewritten as

$$
\sum_{r^{\prime} \in V}\left[\tilde{G}^{0}\left(r, r^{\prime}, \omega\right)-s I\right] \tilde{E}(r)=\frac{-\tilde{E}^{0}(r)}{\varepsilon_{s}(\boldsymbol{r}, \omega)},
$$

where $s=\frac{1}{\varepsilon(\boldsymbol{r}, \omega)-\varepsilon_{0}(\omega)} \cdot \tilde{G}^{0}$, a $3 N \times 3 N$ matrix, is called the Green's matrix. Because $\tilde{G}^{0}$ is symmetrical, mathematically, there are $3 N$ real eigenvalues. Physically, only those eigenvalues with the large residue of electric field correspond to the strong resonances, and the cooperation of positive permittivity (here $\varepsilon_{0}=1.0$ ) and negative permittivity leads to the plasmonic resonances.

In the clusters subspace, the electric field $\tilde{E}(r)$, which is a $3 N \times 1$ matrix, has the general form of [39]

$$
\tilde{E}(r)=\sum_{n=1}^{3 N} A_{n}(s) \cdot R_{n}
$$


where $R_{n}$ is the right eigenvector of the $n$th eigenvalue of $\tilde{G}^{0}$. By substituting eq. (6) into eq. (5) and multiplying by the left eigenvector $L_{n}\left(=R_{n}{ }^{\mathrm{T}}\right), \quad \tilde{E}(r)$ reads

$$
\tilde{E}(r)=\sum_{n=1}^{3 N} \frac{s L_{n} \cdot \tilde{E}^{0}(r)}{s-s_{n}} \cdot R_{n} .
$$

For any point $\boldsymbol{r}$ outside the nanoclusters, we have

$$
E(\boldsymbol{r})=E^{0}(\boldsymbol{r})+k^{2} \sum_{n=1}^{3 N} \frac{\boldsymbol{L}_{n} \cdot \tilde{\boldsymbol{E}}^{0}(r)}{s-s_{n}} \cdot\left[\sum_{r^{\prime} \in C} \boldsymbol{G}^{0}\left(\boldsymbol{r}, \boldsymbol{r}^{\prime}, \omega\right) \cdot \boldsymbol{R}_{n}\right] .
$$

Hence the near field as well as the far field can be analytically expressed.

Without loss, when $s$ approaches one of the eigenvalues $\mathrm{s}_{n}$, i.e. $s \rightarrow \mathrm{s}_{n}$, the electric fields diverge to infinity. Those eigenstates (or resonances) with the strong near field are meaningful and have some applications $[8,10-13,16,17,42]$. To select those resonances, we define the resonance capacity of each $\mathrm{s}_{n}$ in view of the electric field energy inside the nanostructures as [39]

$$
C_{n}=\frac{\int_{V} \mathrm{~d} \boldsymbol{r}^{\prime}\left|\varepsilon_{n}\right| \cdot\left[\operatorname{res}\left|E\left(\boldsymbol{r}^{\prime}\right)\right|\right]_{n}^{2}}{\int_{V} \mathrm{~d} \boldsymbol{r}^{\prime}\left|\varepsilon_{0}(\omega)\right| \cdot\left|E^{0}\left(\boldsymbol{r}^{\prime}\right)\right|^{2}}
$$

with the residue of field $\left[\operatorname{res}\left|E\left(r^{\prime}\right)\right|\right]_{n}=\left|\frac{\boldsymbol{L}_{n} \cdot E^{0}\left(\boldsymbol{r}^{\prime}\right)}{\varepsilon_{n}(\boldsymbol{r}, \omega)} \cdot \boldsymbol{R}_{n}\right|$ and $\varepsilon_{n}=\frac{1}{s_{n}}+\varepsilon_{0}(\omega)$. The value of $C_{n}$ quantitatively expresses the ability of free electrons in metal to gather electromagnetic energy from the environment. By means of the resonance capacity, we can not only select those resonances with strong near fields, but also quantitatively compare the resonance abilities among various subwavelength structures. Conceptually, the extinction peaks of far fields correspond to those resonances with high resonance capacities.

The GMM can be easily extended to a binary nanostructure system [30]. When an arbitrarily shaped binary structure with dielectric permittivities $\varepsilon_{1}(\boldsymbol{r}, \omega)$ and $\varepsilon_{2}(\boldsymbol{r}, \omega)$ is embedded in a homogeneous bulk material $\varepsilon_{0}(\omega)$, the tensors become $\varepsilon_{s i}(\boldsymbol{r}, \omega)\left(=\varepsilon_{i}(\boldsymbol{r}, \omega)-\varepsilon_{0}(\boldsymbol{r}, \omega)\right.$ for $i=1$ or 2$)$ and vanish outside the structures. Via the same transformations, the Green's matrix becomes [30]

$$
\begin{aligned}
\tilde{\boldsymbol{G}}^{0}\left(\boldsymbol{r}, \boldsymbol{r}^{\prime}, \omega\right)= & \delta V k^{2} \boldsymbol{G}^{0}\left(\boldsymbol{r}, \boldsymbol{r}^{\prime}, \omega\right) \delta\left(\boldsymbol{r}^{\prime}-\boldsymbol{r}_{1}\right) \\
& +\eta \delta V k^{2} G^{0}\left(\boldsymbol{r}, \boldsymbol{r}^{\prime}, \omega\right) \delta\left(\boldsymbol{r}^{\prime}-\boldsymbol{r}_{2}\right)
\end{aligned}
$$

for $r_{1} \in C_{1}$ and $r_{2} \in C_{2}$ with $\eta=\left(\varepsilon_{2}-\varepsilon_{0}\right) /\left(\varepsilon_{1}-\varepsilon_{0}\right)$, and eq. (2) becomes

$$
\sum_{\boldsymbol{r}^{\prime} \in C}\left[\tilde{G}^{0}\left(\boldsymbol{r}, \boldsymbol{r}^{\prime}, \omega\right)-s \boldsymbol{I}\right] \cdot \tilde{\boldsymbol{E}}(\boldsymbol{r})=\frac{-\tilde{\boldsymbol{E}}^{0}(\boldsymbol{r})}{\varepsilon_{s_{1}}(\boldsymbol{r}, \omega)},
$$

where $s=\frac{1}{\varepsilon_{s 1}(r, \omega)}=\frac{1}{\varepsilon_{1}(r, \omega)-\varepsilon_{0}(\omega)}$ is the eigenvalue of
$\tilde{G}^{0}\left(\boldsymbol{r}, \boldsymbol{r}^{\prime}, \omega\right)$. For any point $r$ outside the nanoclusters, $E(\boldsymbol{r})$ reads [30]

$$
\begin{aligned}
E(\boldsymbol{r})= & E^{0}(\boldsymbol{r})+k^{2} \sum_{n=1}^{3 N} \frac{\boldsymbol{L}_{n} \cdot \tilde{\boldsymbol{E}}^{0}(r)}{s-s_{n}} \\
& \times\left[\left(\sum_{r^{\prime} \in C_{1}} \boldsymbol{G}^{0}\left(\boldsymbol{r}, \boldsymbol{r}^{\prime}, \omega\right)+\eta \sum_{r^{\prime} \in C_{2}} \boldsymbol{G}^{0}\left(\boldsymbol{r}, \boldsymbol{r}^{\prime}, \omega\right)\right) \cdot \boldsymbol{R}_{n}\right] .
\end{aligned}
$$

When $s$ approaches one of the eigenvalues $s_{n}$, i.e. $s \rightarrow s_{n}$, in binary nanostructures, for each $s_{n}$, the joint resonance capacity reads [30]

$$
C_{n}=\frac{\int_{C_{1}} \mathrm{~d} \boldsymbol{r}^{\prime}\left|\varepsilon_{1 n}\right| \cdot\left[\operatorname{res}\left|E\left(\boldsymbol{r}^{\prime}\right)\right|\right]_{n}^{2}+\int_{C_{2}} \mathrm{~d} \boldsymbol{r}^{\prime}\left|\varepsilon_{2 n}\right| \cdot\left[\operatorname{res}\left|E\left(\boldsymbol{r}^{\prime}\right)\right|\right]_{n}^{2}}{\int_{C} \mathrm{~d} \boldsymbol{r}^{\prime}\left|\varepsilon_{0}(\omega)\right| \cdot\left|E^{0}\left(\boldsymbol{r}^{\prime}\right)\right|^{2}},
$$

which has the same physical meaning as discussed in the one-component nanostructure.

\section{Plasmonic structure design}

Using Green's matrix method and the resonance capacity, the experimental result of nanoantennas has been successfully reproduced $[13,39]$. The resonance capacity for a certain structure-type can be obtained, which makes it possible to tailor the plasmonic properties in the selected wavelength. We have explored the rectangular-type subwavelength metallic structures $(l<\lambda / 15)$ at the wavelength $\lambda=$ $632.8 \mathrm{~nm}$ [39]. Figure 1 displays the resonance capacity distributions of different rectangular structures and polarized fields. For a $28 \mathrm{~nm} \times 28 \mathrm{~nm} \times 28 \mathrm{~nm}$ cube, there are 6 main resonance peaks in Figure 1(b), roughly in line with the results of dipole-dipole approximation [43]. If there is a large spatial extension in the field polarized direction and squeezing in the perpendicular direction, resonance positions are then shifted to the region $s \in(-0.2,0.0)$ (to which the dielectric constants of noble metals can have access) and only one main peak exists. The immunity of resonance capacity distribution to the wavelength in the sub-wavelength structures is also discussed. On the one hand, for a definite structure, its resonance capacity distribution does not vary with the volume zoomed in or out in a quasistatic situation $l$ $<\lambda$. On the other hand, resonance capacity distribution varies only slightly with increasing incident wavelength. These points are very helpful in designing plasmonic nanostructures.

Great efforts have been made seeking plasmonic geometries that are widely tunable at the resonance wavelength $[9,19,44]$ and that have large near field enhancement $[45,46]$. When the size of nanostructure is large enough to break through the quasistatic limit, its resonance capacity will be greatly modified. The dependence of resonance wavelength $\lambda_{R}$ on the geometry of cross section in gold 


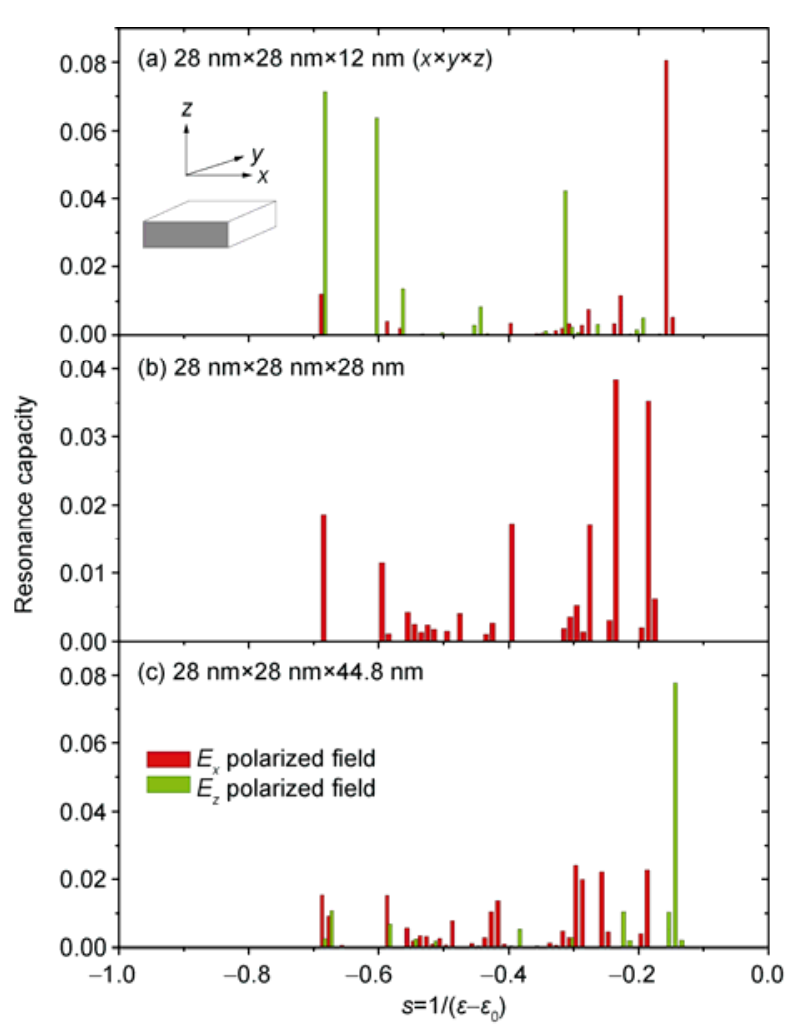

Figure 1 Resonance capacity of a set of rectangular subwavelength structures at $632.8 \mathrm{~nm}$ with $E_{x}$ polarized field and $E_{z}$ polarized field [39]. Here the fields propagate along the $y$ axis.

nanostrips indicates that the resonance wavelength of nanostrip is blue-shifted when the size perpendicular to the polarization direction becomes large. To understand this phenomenon, note that when a surface plasmon resonance happens, a thicker metallic nanostructure will draw more fields into the metallic part from the dielectric environment; then the resonance wavelength of a thicker nanostructure shifts to a shorter wavelength [47]. We consider a p-polarized plane wave (propagating in the $x$ - $y$ plane, polarized along $x$-axis) to be incident at a nanostrip of $l \times w \times h$ (length $l$ along $x$-axis, width $w$ along $y$-axis, and height $h$ along $z$-axis) [21]. The dielectric permittivity parameters of noble metals are available from [48] and the dielectric constant outside the nanostrip is 1. In Figure 2, a set of calculations are performed by changing the gold nanostrip length from $30 \mathrm{~nm}$ to about $120 \mathrm{~nm}$. The dipolar resonance wavelength of nanostrip is red-shifted when the length becomes large. Plasmon resonances can cover almost the entire visible to the near-infrared range. When the cross section is small, the resonance wavelength varies quickly as the length increases, especially in the infrared range. Therefore, to achieve a larger tunability, the nanostrips with smaller cross sections are preferred.

The nanostrip dimensions can vary from several tens of nanometers to several hundred nanometers, so we use the nomenclature $30 \times 10 \times 10$ series to refer to the nanostrips

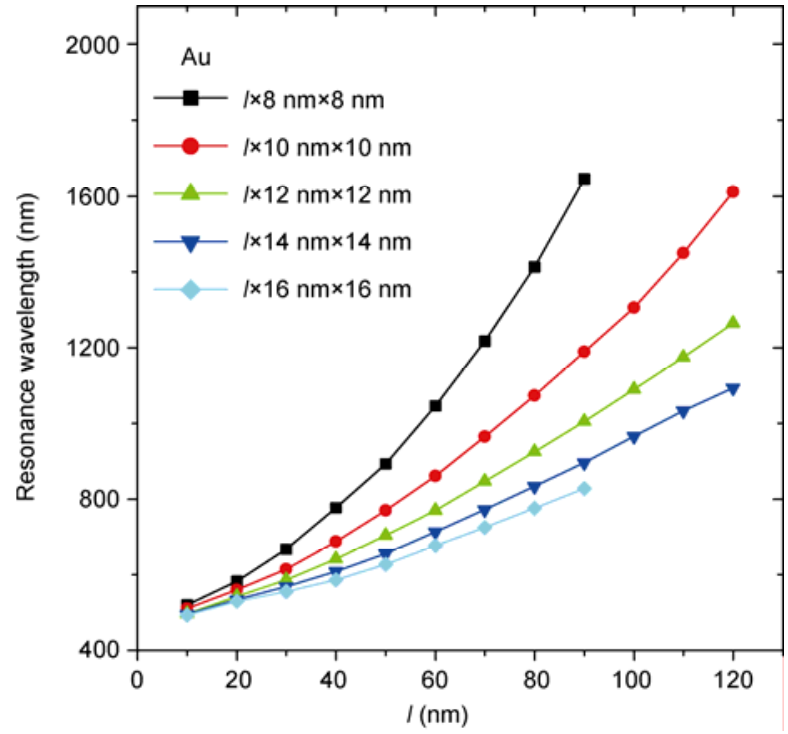

Figure 2 Calculated resonance wavelengths of gold nanostrips as a function of length and for different cross sections. Here the fields propagate perpendicular to the long edge $l$ of nanostrips and are polarized parallel to this edge [21].

with $l: w: h=30: 10: 10$. We find a linear tunability of the resonance wavelength by zooming in or out on one nanostrip, as shown in Figure 3(a) [21]. When the nanostrip size is much smaller than the resonance wavelength, $\lambda_{R}$ varies slowly due to the quasistatic limit [39]. Beyond this limit, with the size increasing proportionally, $\lambda_{R}$ shifts to longer wavelength quickly. In prticular, a good linear tunability is achieved from about $\lambda_{R}=900 \mathrm{~nm}$ to $\lambda_{R}=1800 \mathrm{~nm}$ with the length ranging from $l=210 \mathrm{~nm}$ to $l=600 \mathrm{~nm}$, where the relationship between $\lambda_{R}$ and $l$ can be fitted in a linear way: $\lambda_{R}=a+b \times l$. Roughly speaking, we can conclude that a $1 \mathrm{~nm}$ change in the nanostrip length produces a shift of $2.6 \mathrm{~nm}$ in the resonance wavelength after $R=900 \mathrm{~nm}$. In addition to gold, the linear relations can also apply to silver and copper materials, as shown in Figure 3(b). This linear relation can be intuitively explained as follows. Beyond the electrostatic limit, the real part of dielectric constants meets the SPR changes as the nanostrip size increases; meanwhile, the real part of dielectric constants for real metals changes with incident wavelength, as shown in the inset of Figure 3(b). The accordance of these two change rates results in a linear relationship.

\section{Plasmon control through binary metallic nanostrcutures}

The SPR properties of two closely packed metallic nanoparticles with the same geometry and the same dielectric permittivity have been widely investigated [24-29]. When the central line of both particles is parallel to the polarization of the electric field, the resonance wavelength red shifts and when the central line of both particles is perpendicular to 

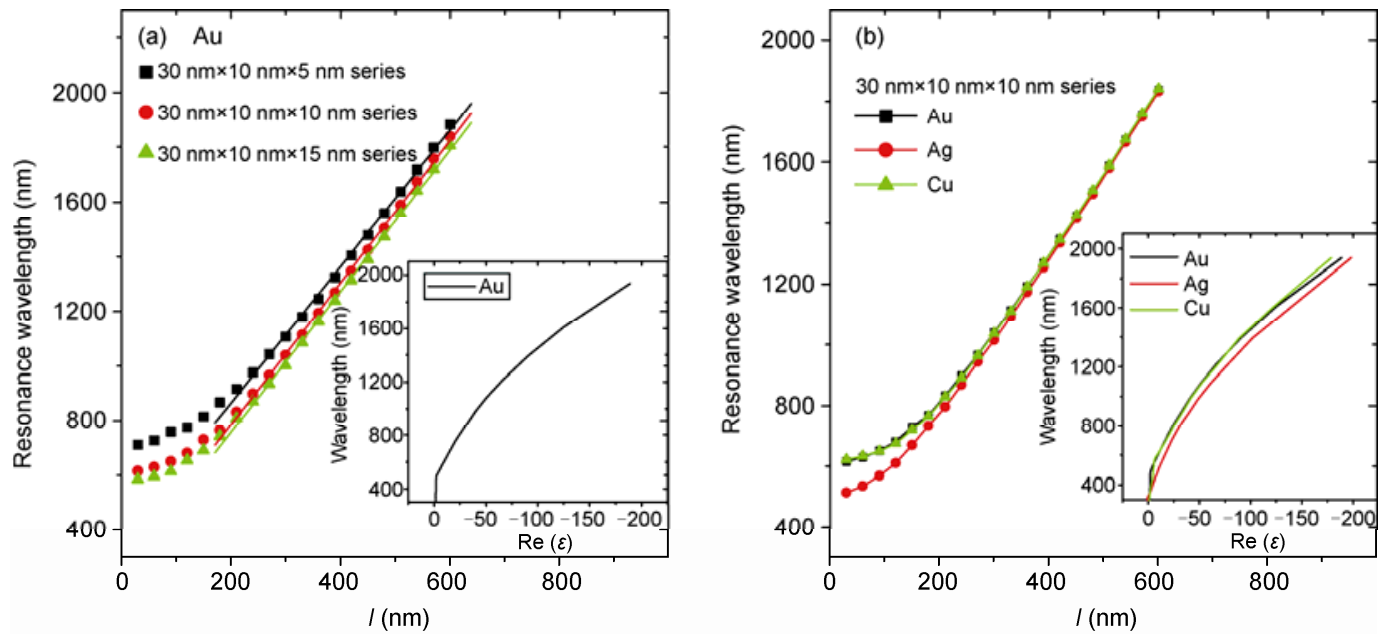

Figure 3 Calculated resonance wavelengths of gold nanostrips with three aspect ratios (the solid lines are linear fits to the data points from $l=210 \mathrm{~nm}$ to $l$

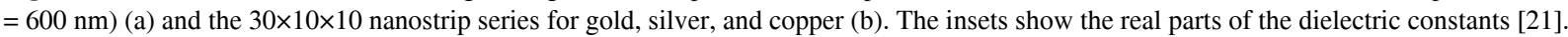

the polarization of the electric field, a blue-shift occurs. It is known that the resonance occurs at the matching conditions of the geometry, incident light, metallic permittivity and dielectric environment. Thus, the position and intensity of the resonances can be affected by neighboring nanoparticles. By introducing the difference permittivity ratio $\eta=\left(\varepsilon_{2}-\varepsilon_{0}\right) /\left(\varepsilon_{1}-\varepsilon_{0}\right)$, the GMM for computing surface plasmon resonances is extended to binary nanostructures [30]. Based on the near field coupling, the interplay of plasmon resonances between two closely packed nanostrips is investigated. At a fixed wavelength, with the varying $\eta$, the resonances exhibit different regions: the dielectric effect region, the resonance chaos region, the collective resonance region, the resonance flat region, and the new branches region [30]. Simultaneously, the avoiding crossing and mode transfer phenomena between the resonance branches are observed. These findings are helpful to controlling the plasmonic resonances of subwavelength structures.

As shown in Figure 4(a), when the central line of both nanostrips is parallel to the polarization of the external electric field, we call it the parallel case, whereas when the central line is perpendicular to the polarization of the electric field, we call it the perpendicular case. Here the input wavelength is $\lambda=632.8 \mathrm{~nm}$, the size of each nanostrip is 60 $\mathrm{nm} \times 20 \mathrm{~nm} \times 20 \mathrm{~nm}$, and both gaps are $10 \mathrm{~nm}$ with a $5 \mathrm{~nm}$ discretization mesh. The incident electric field is propagating along the $z$ direction and polarized along the $x$ direction. With calculations of the near field distributions associated with resonances, we have selected the $x y$ plane $10 \mathrm{~nm}$ above the nanostrips and included imaginary parts of dielectric constants of $0.15 \mathrm{i}$ in the parallel case and $0.05 \mathrm{i}$ in the perpendicular case. At $\lambda=632.8 \mathrm{~nm}$, for a single $60 \mathrm{~nm} \times 20$ $\mathrm{nm} \times 20 \mathrm{~nm}$ nanostrip, there is only one resonance at $\varepsilon_{1}(\omega)$ $\approx-11.7$, which corresponds to the gold material [39].

Now let us outline the main results of the parallel case [30], and clarify the mode classification and the nomination of resonance branches in the $s-\eta$ diagrams in Figure 4. Four main branches represent 4 types of resonances associated with nanostrip 1 , whose intensities are expressed as $C_{1}$. When $\eta<0$, i.e. nanostrip 2 is a dielectric being closely located to the resonant nanostrip 1, resonances are slightly affected by the dielectric nanoparticle. Thus, this is the dielectric effect region, flagged as branch 1 . However, when nanostrip 2 is metallic, i.e. $\eta>0$, there will be a large influence on the resonant properties of metallic nanostrip 1 due to the existence of free electrons in metallic nanostrip 2. When $\eta \in[0,0.95]$, the resonance capacity of nanostrip 1 is very sensitive to $\eta$, which is called the resonance chaos region, marked as subbranch 21 . For $\eta \in[0.65,1.3]$, i.e. when two metallic nanostrips have comparable dielectric permittivities, collective resonances occur and this is called the collective resonance region, marked as subbranch 22 . When $\eta>1.5$, the resonance capacity becomes large and stable, called the resonance flat region and marked as subbranch 23 . Finally, when $\eta>3.0$, the new branches 3 and 4 appear, called new branch region. The details of each region are given below [30].

Dielectric effect region. In this region, resonances are dominated by nanostrip 1 and only marginally affected by the dielectric nanostrip 2 because the electromagnetic wave can penetrate the dielectric. At approximately $\eta=-1.56$, there is an avoiding crossing between subbranches 11 and 12 in Figure 4(b) and we observe a minimum resonance capacity in Figure 4(c). Simultaneously, near $\eta=-1.56$, when the branch 11 is transferred to branch 12 , the splitting of SPR is also found, i.e. for one $\eta$, there are two close large resonances. Near field distributions around the area $A$ are illustrated in Figure 5(a). With $\eta$ decreasing from -1.5 to -1.6 , there appears a depression in the intensity of near fields at $\eta=-1.56$ and the near fields are localized around the nanostrip 1.

Resonance chaos region. It is flagged as subbranch 21 . Now the resonance capacity of nanostrip 1 is very sensitive 

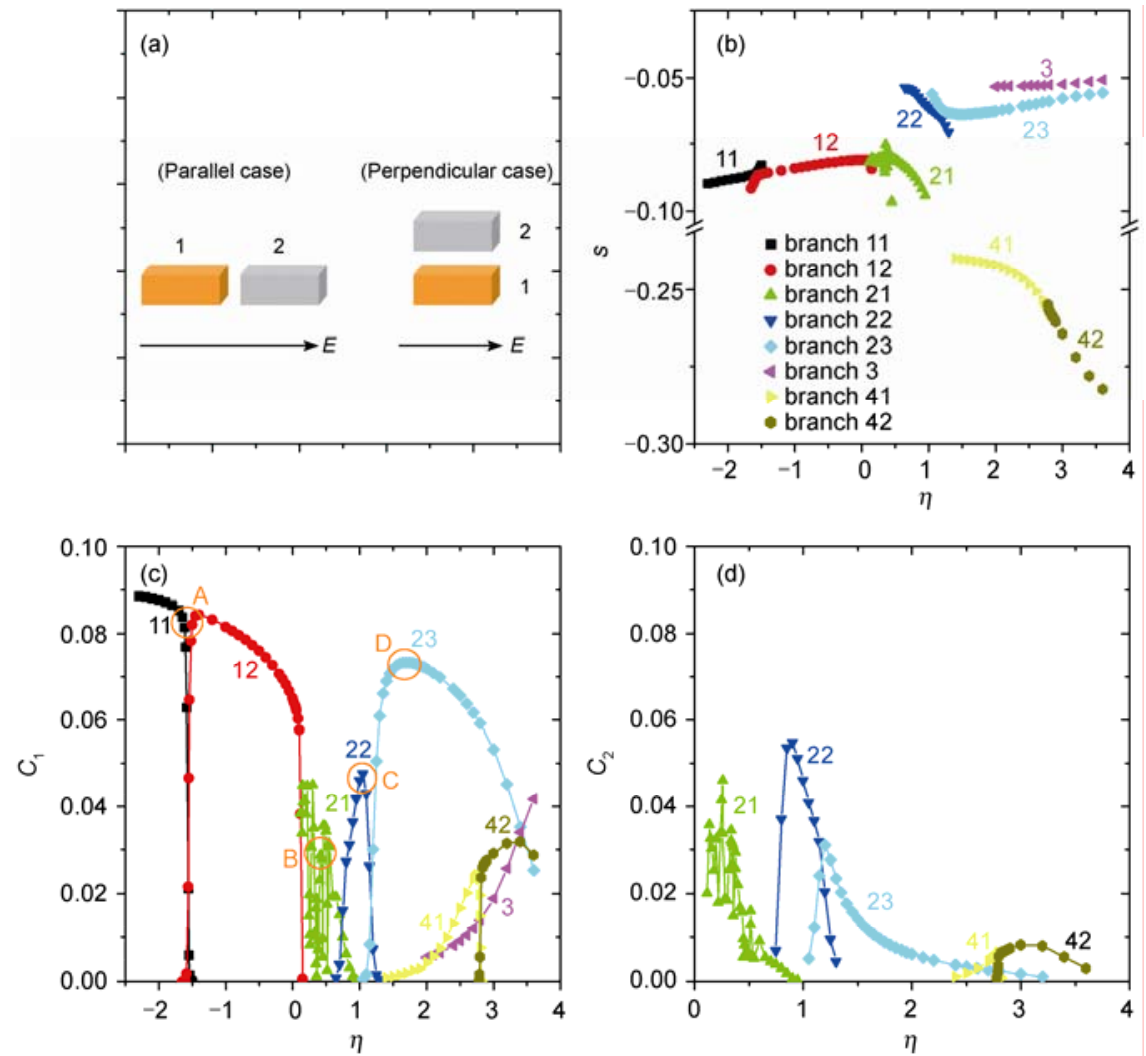

Figure 4 (a) Scheme of two $60 \mathrm{~nm} \times 20 \mathrm{~nm} \times 20 \mathrm{~nm}$ nanostrips for parallel and perpendicular cases. Resonance combination for the parallel case with varying $\eta$ : (b) resonance branches $s$, (c) resonance capacity for nanostrip 1 and (d) resonance capacity for nanostrip 2 [30].

to $\eta$; although we could not find explicit features, the resonances are clearly dominated by nanostrip 1 under the condition $\left|\varepsilon_{1}\right|>\left|\varepsilon_{2}\right|$. This "chaotic" phenomenon may result from the very high density of branches. It is not easy to classify each resonance subbranch and we have only selected those with the strongest resonances. Figure 5(b) illustrates the near field distributions near the area $B$ at $\eta=-0.30,0.36$, and 0.40 , and the near field sensitivity to $\eta$ is clearly shown.

Collective resonance region. When the dielectric permittivities of two nanostrips have comparable values, the following characterization of the collective resonances can be made. That is subbranch 22 . When $\eta=1, \varepsilon_{1}=\varepsilon_{2}=$ -15.32 , if the permittivity region is mapped to the wavelength region, compared with the result of a single nanostrip, there is a large red-shift in the resonance frequency [24-27]. The detailed near field distributions around area $C$ are illustrated in Figure 5(c). It is noted that for $\eta=1$ the strong near fields are distributed outside of the two nanostrips, rather than in the center of them. If we choose the $X Y$ plane within the metal, the electric field is enhanced within the gap, in line with the nanoantenna results [13].

Resonance flat region. Following the collective resonance region, the resonance enters the resonance flat region, marked as branch 23. We have found that, though $\left|\varepsilon_{1}\right|<\left|\varepsilon_{2}\right|$, the resonances of nanostrip 1 dominate the system. This is different from what was found in branch 21 , where $\left|\varepsilon_{1}\right|>\left|\varepsilon_{2}\right|$ and the nanostrip 1 dominates. Figure 5(d) illustrates the near field distributions around the area $D$.

New resonance branches. When $\eta>3.0$, near branch 23, the new branches 3 and 4 emerge. Branch 23 disappears and the main resonant behavior is determined by branches 3 and 4. In particular, at $\eta=3.4$, the resonance capacities of surface plasmon in branch 23, branch 3 and branch 4 have almost the same values, exhibiting resonance splitting or multiple resonance phenomena. Previously, the multiple resonance of surface plasmon has been reported in the nanocrescent and C-type structures [49,50]. It is found that near $\eta=2.8$ the avoiding crossing and mode transfer phenomena between branch 41 and branch 42 appear. We note as well that within the quasistatic limit the same phenomenon of dielectric resonance occurs [51,52].

Except for the disordered subbranch 21, for one subbranch, there is always a smooth curve of resonance capacity that starts from zero, passes the maximum value, and finally reaches to zero again; and typically one branch is up and another branch down, as shown in Figure 4(c). For a large difference between both permittivities, we observe that generally one particle dominates the resonance. However, it is not always the metallic particle with the largest permittivity that determines the resonances. Sometimes, the nanoparicle with the smallest permittivity dominates. While, in the perpendicular case, there are about 4 main branches, 

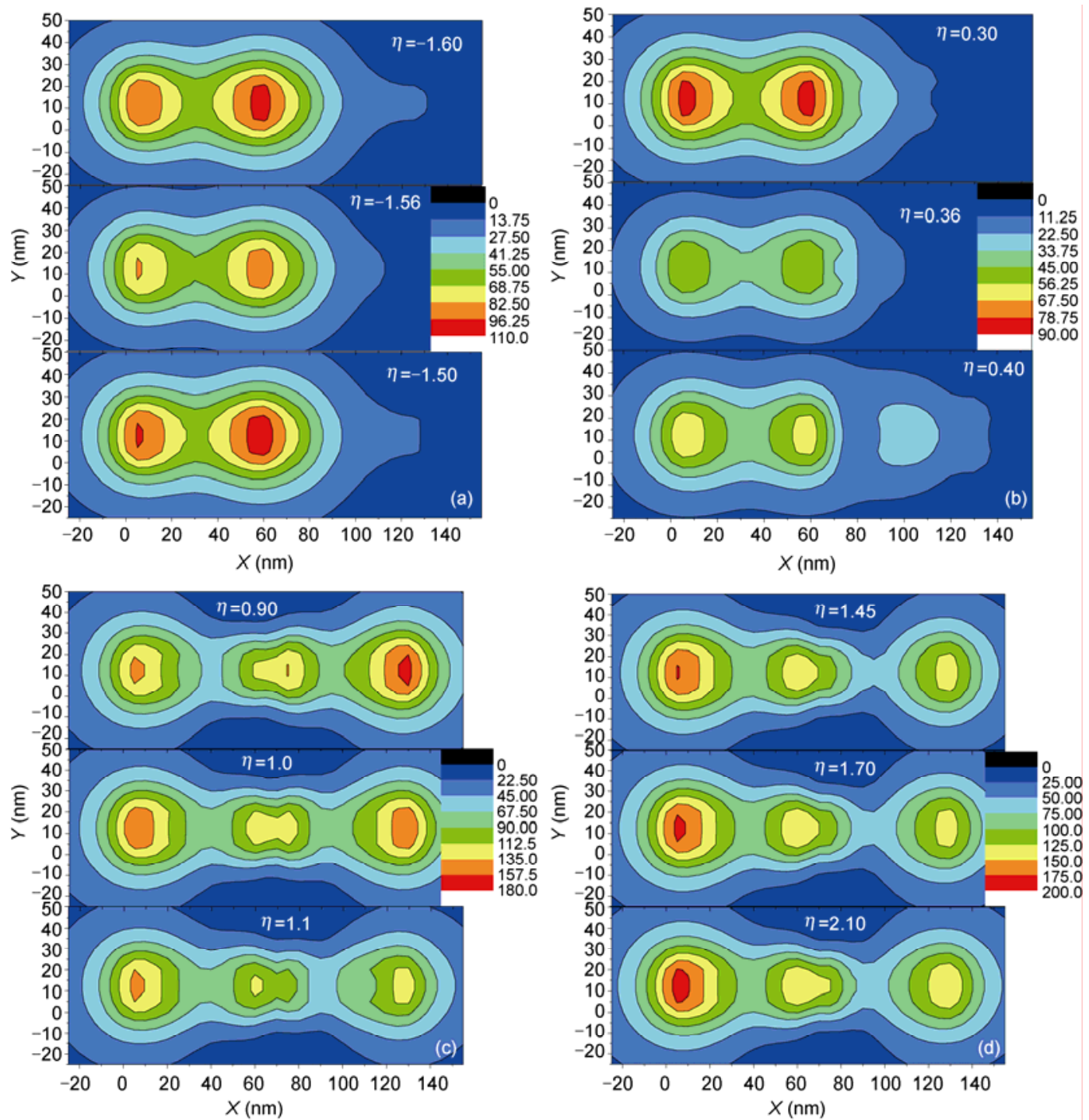

Figure 5 Near field distributions above $10 \mathrm{~nm}$ of nanostrips around area $A$ (a), area $B$ (b), area $C$ (c), and area $D(\mathrm{~d})$ [30].

but it is very difficult to distinguish those subbranches. Branch 1 is still the dielectric effect region, branch 2 roughly includes the resonance chaos region, collective resonance region, and resonance flat region, and branches 3 and 4 belong to the new branches regions. Compared with the parallel case, the resonance behavior of the perpendicular case is more complicated [30].

Investigation on the interplay of plasmonic resonances reveals that plasmonic resonances can be controlled by material parameters $\varepsilon_{1}$ and $\varepsilon_{2}$. For binary three-strip nanostructures, near fields within the gaps and at the ends of nanostrips are greatly enhanced as a result of the influence of neighboring metallic material. Then, along each resonance branch, resonances in the dielectric permittivity region are mapped to the wavelength region of gold [31]. By adjusting material parameters $\varepsilon_{1}$ and $\varepsilon_{2}$, the resonance wavelength is tuned from $\lambda_{R}=500 \mathrm{~nm}$ to $\lambda_{R}=1500 \mathrm{~nm}$; whereas for a single nanostrip it is only at $\lambda_{R}=630 \mathrm{~nm}$. We also find that comparable permittivity parameters $\varepsilon_{1}$ (or $\varepsilon_{2}$ ) and $\varepsilon_{\mathrm{au}}(\omega)$ can do a good job of controlling the resonance wavelength and intensity, whereas the high dielectric permittivity of neighboring metal has an advantage of producing a giant enhancement of the near field. These findings will provide new insights into hybrid plasmonic device designs such as for plasmonic sensors.

For binary three-strip nanostructures, gold is used in two cases: (i) Au is used in two outer nanostrips and $\varepsilon_{2}$ in the middle strip, and (ii) $\mathrm{Au}$ is used in the middle nanostrip and $\varepsilon_{1}$ in the two outer strips, the results of which are shown in Figures 6 and 7. Then by adjusting $\varepsilon_{2}$ in case (i) and $\varepsilon_{1}$ in case (ii), plasmonic resonances can be well controlled [31]. We first consider the situation where a dielectric material is used to control the resonances of the metallic material. It is seen in branch 1 of Figures 6(a) and 7(a) and in branch 2 of Figures 6(b) and 7(b) that the resonance wavelength is almost unchanged and the resonance capacity only has a very 

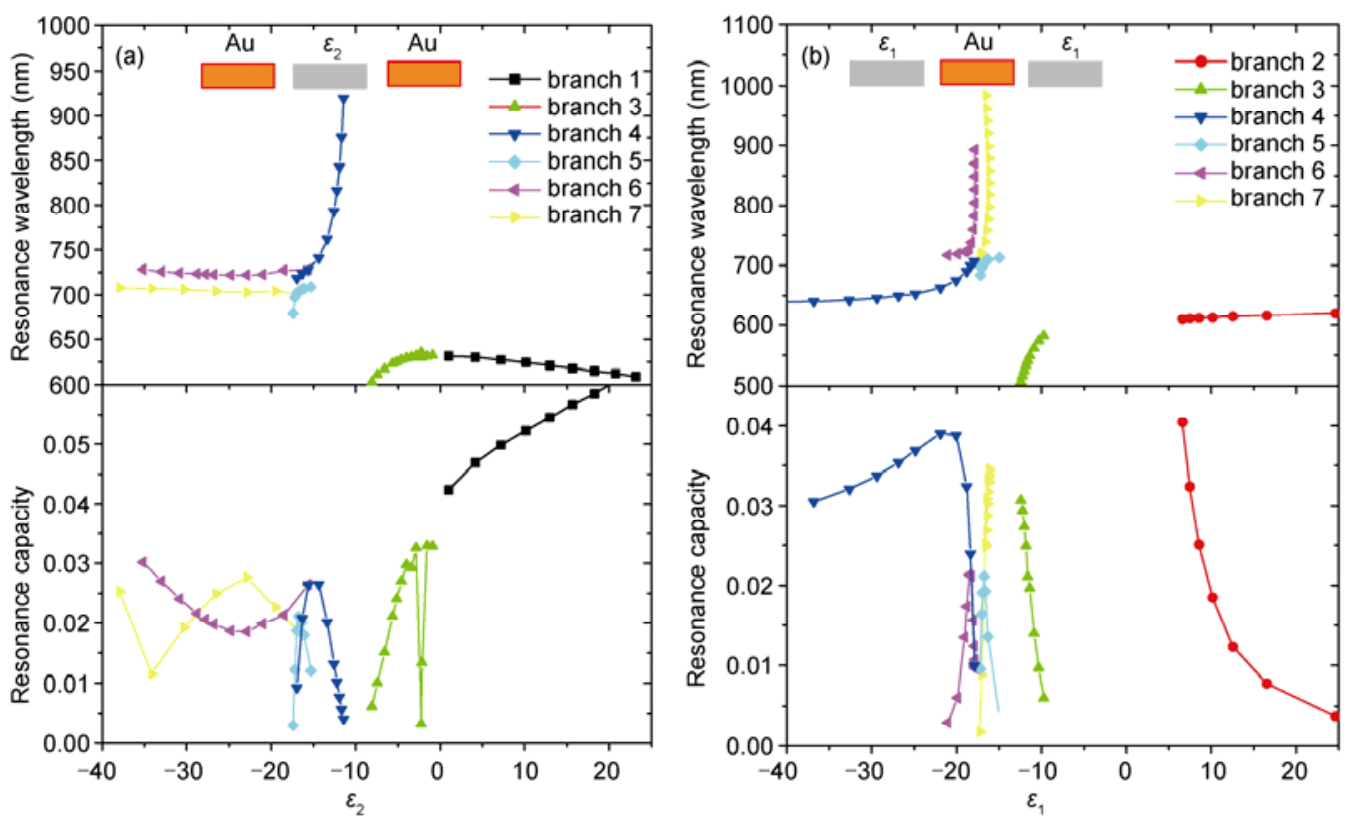

Figure 6 Plasmonic resonances of Au in a parallel binary three-strip nanostructure as a function of $\varepsilon_{2}$ in the middle strip (a) and of $\varepsilon_{1}$ in both outer strips (b) [31].

small modulation with the varying $\varepsilon_{1}\left(\varepsilon_{2}\right)$. Because the electromagnetic wave can penetrate a dielectric, it is not suitable to adjust the plasmonic resonances.

Comparing to $\varepsilon_{\mathrm{au}}(632.8 \mathrm{~nm})=-11.78$, an excessively large value of $\varepsilon_{1}$ (or $\varepsilon_{2}$ ) or an excessively small value of $\varepsilon_{1}$ (or $\varepsilon_{2}$ ) has little effect on the resonance wavelength, but has a large effect on the resonance capacity. In the parallel case, as shown in Figure 6, when $\varepsilon_{2}$ (or $\varepsilon_{1}$ ) belongs to the region $[-40,-20]$, the resonance wavelengths are changed from $\lambda_{R}=700 \mathrm{~nm}$ to $\lambda_{R}=725 \mathrm{~nm}$ (or around $\lambda_{R}=650 \mathrm{~nm}$ ), and the resonance capacity can be modified greatly under the condition $\left|\varepsilon_{2}\right|\left(\right.$ or $\left.\left|\varepsilon_{1}\right|\right)>\varepsilon_{\mathrm{au}}$. For $\varepsilon_{1}\left(\right.$ or $\left.\varepsilon_{2}\right) \in[-10,0]$, the resonance wavelength of $\mathrm{Au}$ and the resonance capacity almost present no visible changes; in Figure 6(b), we cannot find strong resonances in the region. Then the perpendicular case is explored. The same things happen in Figure 7, only
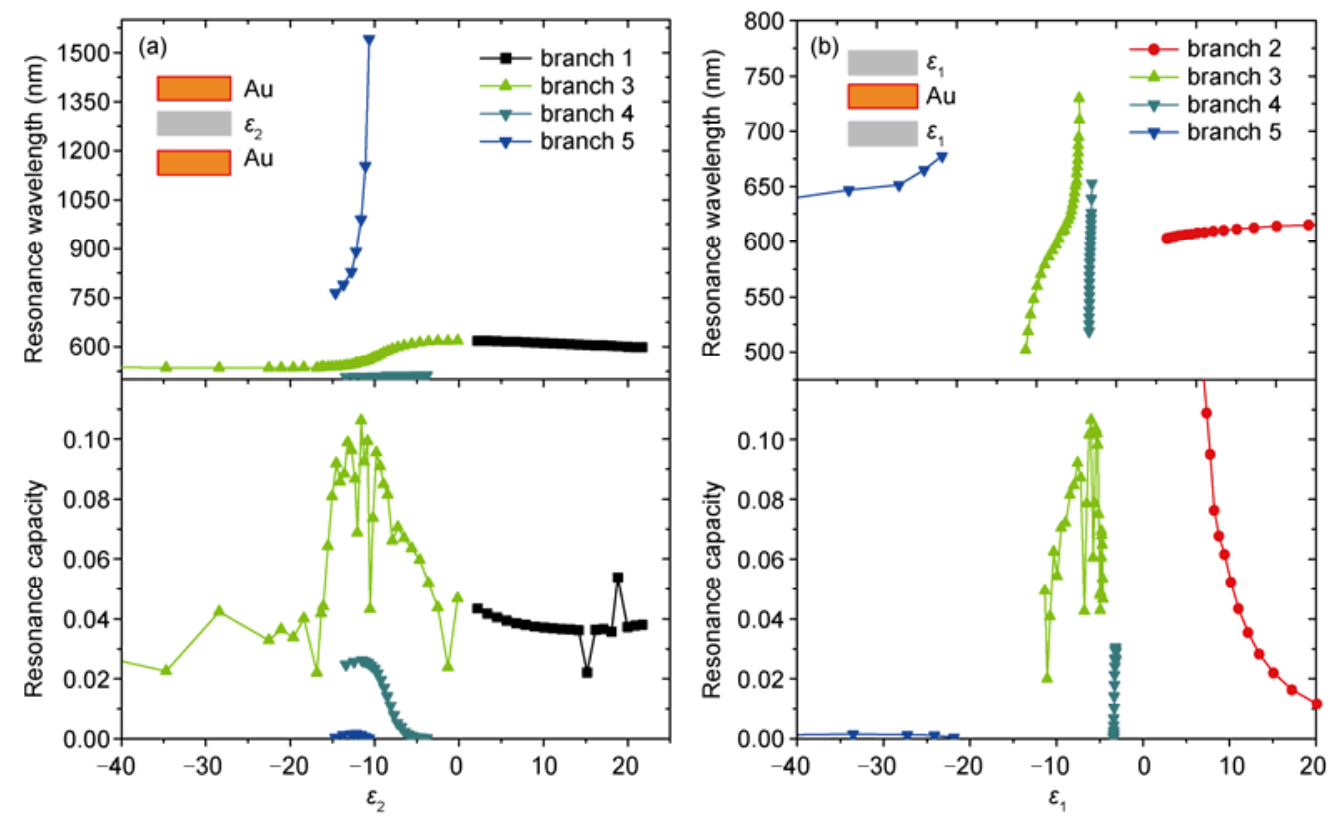

Figure 7 Plasmonic resonances of Au in a perpendicularl binary three-strip nanostructure as a function of $\varepsilon_{2}$ in the middle strip (a) and $\varepsilon_{1}$ in both outer strips (b) [31]. 
with different parameter regions. When $\varepsilon_{2}$ (or $\left.\varepsilon_{1}\right) \in[-40$, -15], the resonance wavelength is changed around $\lambda_{R}=630$ $\mathrm{nm}$ (or from $\lambda_{R}=650 \mathrm{~nm}$ to $\lambda_{R}=675 \mathrm{~nm}$ ), and there is a comparable small resonance capacity under the condition $\left|\varepsilon_{2}\right|\left(\right.$ or $\left.\left|\varepsilon_{1}\right|\right)>\left|\varepsilon_{\mathrm{au}}\right|$. Finally, for $\varepsilon_{1}$ (or $\left.\varepsilon_{2}\right) \in[-5,0]$, the resonance wavelength and resonance capacity almost present no change. The region where the permittivity parameter $\varepsilon_{1}$ (or $\left.\varepsilon_{2}\right)$ is comparable with $\varepsilon_{\mathrm{au}}(\omega)$ is discussed. In the parallel case as shown in Figure 6, when $\varepsilon_{1}$ (or $\left.\varepsilon_{2}\right) \in[-20,-10]$, the resonance wavelength of Au can be tuned in a wide spectral region from $\lambda_{R}=650 \mathrm{~nm}$ to $\lambda_{R}=1000 \mathrm{~nm}$, and multi-branch resonances coexist due to a strong interplay between two different materials. This multi-resonance phenomenon has some applications in the plasmonic device design $[49,50]$. In the perpendicular case, at about $\varepsilon_{1}$ (or $\left.\varepsilon_{2}\right) \in[-15,-5]$, and almost the same features of resonance branches are found, namely, the resonance wavelength can be tuned from $\lambda_{R}=500 \mathrm{~nm}$ to $\lambda_{R}=1500 \mathrm{~nm}$, the resonance capacity has a large increment, and multi-branch resonances coexist. It is noted in Figure 7 that the resonance wavelength of $\mathrm{Au}$ can reach to $\lambda_{R}=1500 \mathrm{~nm}$. Therefore we may conclude that, for this kind of binary three-strip nanostructure, the resonance wavelength and intensity can be well modulated in the region where the permittivity parameter $\varepsilon_{1}$ (or $\varepsilon_{2}$ ) can be competitive with $\varepsilon_{\mathrm{au}}(\omega)$.

\section{Summary}

We have reviewed the Green's matrix method of solving the plasmonic resonances and its applications to the plasmonic structure design and control. The well-designed nanostrips have realized a tunability from the visible to near-infrared in spectrum. In particular, a linear relation has been found between the nanostrip size and the dipolar resonance wavelength. When both nanostructures are metal, the resonance properties are generally dominated by one of the metals or exhibited as a collective resonance of both metals. Through adjusting the material parameters, both the resonance wavelength and the resonance intensity can be well controlled. In principle, the above phenomena could be probed by the scanning near-field optical microscope technique [53-56]. The method provides a powerful tool for plasmonic optics, such as for the design of subwavelength plasmonic structures, the guidelines for nanostructure-assisted fluorescence, SERS, and sensing, and the foundation of the design of hybrid plasmonic devices.

This work was supported by the National Natural Science Foundation of China $(10874004,10674009,10821062)$ and National Basic Research Program of China (2007CB307001).

1 Girard C, Dereux A. Near-field optics theories. Rep Prog Phys, 1996, 59: $657-699$
2 Girard C, Joachim C, Gauthier S. The physics of the near-field. Rep Prog Phys, 2000, 63: 893-938

3 Boardman A D. Electromagnetic Surface Modes. New York: John Wiley and Sons, 1982

4 Raether H. Surface Plasmons. Berlin: Springer, 1988

5 Zayats A V, Smolyaninov I I, Maradudin A A. Nano-optics of surface plasmon polaritons. Phys Rep, 2005, 408: 131-314

6 Rather H. Surface Plasmons on Smooth and Rough Surfaces and on Gratings. Berlin: Springer, 2004

7 Kottmann J P, Martin O J F, Smith D R, et al. Plasmon resonances of silver nanowires with a nonregular cross section. Phys Rev B, 2001, 64: 235402

8 Wokaun A, Gordon J P, Liao P F. Radiation damping in surface-enhanced Raman scattering. Phys Rev Lett, 1982, 48: 957-960

9 Lal S, Link S, Halas N J. Nano-optics from sensing to waveguiding. Nat Photonics, 2007, 1: 641-648

10 Maier S A, Kik P G, Atwater H A. Observation of coupled plasmonpolariton modes in Au nanoparticle chain waveguides of different lengths: Estimation of waveguide loss. Appl Phys Lett, 2002, 81: 1714-1716

11 Mitsui K, Handa Y, Kajikawa K. Optical fiber affinity biosensor based on localized surface plasmon resonance. Appl Phys Lett, 2004, 85: 4231-4233

12 Wang T J, Lin W S. Electro-optically modulated localized surface plasmon resonance biosensors with gold nanoparticles. Appl Phys Lett, 2006, 89: 173903

13 Mhlschlegel P, Eisler H J, Martin O J F, et al. Resonant optical antennas. Science, 2005, 308: 1607-1609

14 Cole J R, Halas N J. Optimized plasmonic nanoparticle distributions for solar spectrum harvesting. Appl Phys Lett, 2006, 89: 153120

15 Catchpole K R, Polman A. Plasmonic solar cells. Opt Express, 2008, 16: $21793-21800$

16 Wokaun A, Bergman J G, Heritage J P, et al. Surface second-harmonic generation from metal island films and microlithographic structures. Phys Rev B, 1981, 24: 849-856

17 Kim E M, Elovikov S S, Murzina T V, et al. Surface-enhanced optical third-Harmonic generation in Ag island films. Phys Rev Lett, 2005, 95: 227402

18 Danckwerts M, Novotny L. Optical frequency mixing at coupled gold nanoparticles. Phys Rev Lett, 2007, 98: 026104

19 Murphy C J, Sau T K, Gole A M, et al. Anisotropic metal nanoparticles: Synthesis, assembly, and optical applications. J Phys Chem B, 2005, 109: 13857-13870

20 Kim F, Song J H, Yang P D. Photochemical synthesis of gold nanorods. J Am Chem Soc, 2002, 124: 14316-14317

21 Li J, Gu Y, Zhou F, et al. A designer approach to plasmonic nanostructures: tuning their resonance from visible to near-infrared. J Mod Opt, 2009, 56: 1396-1402

22 Wiley B J, Chen Y C, McLellan J M, et al. Synthesis and optical properties of silver nanobars and nanorice. Nano Lett, 2007, 7: $1032-1036$

23 Lu Y, Liu G L, Kim J, et al. Nanophotonic crescent Moon structures with sharp edge for ultrasensitive biomolecular detection by local electromagnetic field enhancement effect. Nano Lett, 2005, 5: $119-124$

24 Tamaru H, Kuwata H, Miyazaki H T, et al. Resonant light scattering from individual $\mathrm{Ag}$ nanoparticles and particle pairs. Appl Phys Lett, 2002, 80: 1826-1828

25 Gunnarsson L, Rindzevicius T, Prikulis J, et al. Confined plasmons in nanofabricated single silver particle pairs: Experimental observations of strong interparticle interactions. J Phys Chem. B, 2005, 109: 1079-1087

26 Jain P K, Eustis S, El-Sayed M A. Plasmon coupling in nanorod assemblies: Optical absorption, discrete dipole approximation simulation, and exciton-coupling model. J Phys Chem B, 2006, 110: $18243-18253$

27 Reinhard B M, Siu M, Agarwal H, et al. Calibration of dynamic molecular rulers based on plasmon coupling between gold nanoparticles. Nano Lett, 2005, 5: 2246-2252 
28 Jain P K, Huang W Y, El-Sayed M A. On the universal scaling behavior of the distance decay of plasmon coupling in metal nanoparticle pairs: A plasmon ruler equation. Nano Lett, 2007, 7: 2080-2088

29 Jain P K, El-Sayed M A. Universal scaling of plasmon coupling in metal nanostructures: Extension from particle pairs to nanoshells. Nano Lett, 2007, 7: 2854-2858

$30 \mathrm{Gu}$ Y, Wang Y, Li J, et al. Interplay of plasmon resonances in binary nanostructures. Appl Phys B, 2010, 98: 353-363

$31 \mathrm{Gu} \mathrm{Y,} \mathrm{Li} \mathrm{J,} \mathrm{Martin} \mathrm{O} \mathrm{J} \mathrm{F,} \mathrm{et} \mathrm{al.} \mathrm{Controlling} \mathrm{plasmonic} \mathrm{resonances} \mathrm{in}$ binary metallic nanostructures. J Appl Phys, 2010, 107: 114313

32 Taflove A, Hagness S C. Computational Electrodynamics: The Finite-Difference Time-Domain Method. Boston: Artech House, 2005

33 Draine B T, Flatau P J. Discrete-dipole approximation for scattering calculations. J Opt Soc Am A, 1994, 11: 1491-1499

34 Kreibig U, Vollmer M. Optical Properties of Metal Clusters. Berlin: Springer-Verlag, 1995

35 Martin O J F, Girard C, Dereux A. Generalized field propagator for electromagnetic scattering and light confinement. Phys Rev Lett, 1995, 74: 526529

36 Prodan E, Radloff C, Halas N J, et al. A hybridization model for the plasmon response of complex nanostructures. Science, 2003, 302: $419-422$

37 Noguez C. Surface plasmons on metal nanoparticles: The influence of shape and physical environment. J Phys Chem C, 2007, 111: 3806-3819

$38 \mathrm{Gu}$ Y, Yu K W, Sun H. Local-field distribution in resonant composites: Greens-function formalism. Phys Rev B, 1999, 59: 12847-12852

$39 \mathrm{Gu} \mathrm{Y,} \mathrm{Chen} \mathrm{L} \mathrm{L,} \mathrm{Zhang} \mathrm{H} \mathrm{X,} \mathrm{et} \mathrm{al.} \mathrm{Resonance} \mathrm{capacity} \mathrm{of} \mathrm{surface}$ plasmon on subwavelength metallic structures. Europhys Lett, 2008, 83: 27004

40 Morse P M, Feshbach H. Methods of Theoretical Physics. New York: McGraw-Hill, 1953

41 Economou E N. Green's Functions in Quantum Physics. 2nd ed. Berlin: Springer, 1990

42 Danckwerts M, Novotny L. Optical frequency mixing at coupled gold nanoparticles. Phys Rev Lett, 2007, 98: 026104

43 Fuchs R. Theory of the optical properties of ionic crystal cubes. Phys Rev B, 1975, 11: 1732-1740

44 Oldenburg S J, Averitt R D, Westcott S L, et al. Nanoengineering of optical resonances. Chem Phys Lett, 1998, 288: 243-247

45 Hartschuh A, Sanchez E J, Xie X S, et al. High-resolution near-field Raman microscopy of single-walled carbon nanotubes. Phys Rev Lett, 2003, 90: 095503

46 Jackson J B, Westcott S L, Hirsch L R, et al. Controlling the surface enhanced Raman effect via the nanoshell geometry. Appl Phys Lett, 2003, 82: 257-259

47 Wang F, Shen Y R. General properties of local plasmons in metal nanostructures. Phys Rev Lett 2006, 97: 206806

48 Johnson P B, Christy R W. Optical constants of the noble metals. Phys Rev B, 1972, 6: 4370-4379

49 Sheridan A K, Clark A W, Glidle A, et al. Multiple plasmon resonances from gold nanostructures. Appl Phys Lett, 2007, 90: 143105

50 Clark A W, Sheridan A K, Glidle A, et al. Tuneable visible resonances in crescent shaped nano-split-ring resonators. Appl Phys Lett, 2007, 91: 093109

$51 \mathrm{Gu}$ Y, Gong Q H. Avoiding crossing of dielectric resonances in three-component composites. Phys Rev B, 2003, 67: 014209

$52 \mathrm{Gu} \mathrm{Y,} \mathrm{Gong} \mathrm{Q} \mathrm{H.} \mathrm{Localized} \mathrm{mode} \mathrm{transfer} \mathrm{and} \mathrm{optical} \mathrm{response} \mathrm{in}$ three-component composites. Phys Rev B, 2004, 69: 035105

$53 \mathrm{Li}$ Z, Gong Q H. The plasmonic coupling of metal nanoparticles and its implication for scanning near-field optical microscope characterization. Chinese Sci Bull, 2009, 54: 3843-3843

54 Cao Z X, Wu L N. Display technique based on surface plasmon resonant effect. Chinese Sci Bull, 2008, 53: 2257-2264

55 Yao H M, Li Z, Gong Q H. Coupling-induced excitation of a forbidden surface plasmon mode of a gold nanorod. Sci China Ser G-Phys Mech Astron, 2009, 52: 1129-1138

56 Zhao H W, Huang X G, Huang J T. Surface plasmon polaritons based optical directional coupler. Sci China Ser G-Phys Mech Astron, 2008, 51: $1877-1882$ 